\title{
Age- and Sex-Specific Plasticity in Dopamine Transporter Function Revealed by Food Restriction and Exercise in a Rat Activity-Based Anorexia Paradigm $\$$
}

(ㄱ) Lee Gilman, W. Anthony Owens, Christina M. George, Lauren Metzel, Melissa Vitela, Livia Ferreira, (1) Melodi A. Bowman, (1) Georgianna G. Gould, (1)Glenn M. Toney, and (1)Lynette C. Daws

Department of Cellular and Integrative Physiology (T.L.G., W.A.O., C.M.G., L.M., M.V., L.F., M.A.B., G.G.G., G.M.T., L.C.D.), Addiction Research, Treatment \& Training Center of Excellence (T.L.G., L.C.D.), Center for Biomedical Neuroscience (G.M.T., L.C.D.), and Department of Pharmacology (L.C.D.), University of Texas Health Science Center at San Antonio, San Antonio, Texas

Received June 13, 2019; accepted August 21, 2019

\begin{abstract}
Eating disorders such as anorexia typically emerge during adolescence, are characterized by engagement in compulsive and detrimental behaviors, and are often comorbid with neuropsychiatric disorders and drug abuse. No effective treatments exist. Moreover, anorexia lacks adolescent animal models, contributing to a poor understanding of underlying age-specific neurophysiological disruptions. To evaluate the contribution of dopaminergic signaling to the emergence of anorexia-related behaviors during the vulnerable adolescent period, we applied an established adult activity-based anorexia (ABA) paradigm (food restriction plus unlimited exercise access for 4 to 5 days) to adult and adolescent rats of both sexes. At the end of the paradigm, measures of plasma volume, blood hormone levels, dopamine transporter (DAT) expression and function, acute cocaine-induced locomotion, and brain water weight were taken. Adolescents were dramatically more affected by the ABA paradigm than adults in all measures. In vivo chronoamperometry and cocaine locomotor responses revealed sex-specific changes in adolescent DAT function after ABA that were independent of DAT expression differences. Hematocrit, insulin, ghrelin, and
\end{abstract}

corticosterone levels did not resemble shifts typically observed in patients with anorexia, though decreases in leptin levels aligned with human reports. These findings are the first to suggest that food restriction in conjunction with excessive exercise sex-dependently and age-specifically modulate DAT functional plasticity during adolescence. The adolescent vulnerability to this relatively short manipulation, combined with blood measures, evidence need for an optimized ageappropriate ABA paradigm with greater face and predictive validity for the study of the pathophysiology and treatment of anorexia.

\section{SIGNIFICANCE STATEMENT}

Adolescent rats exhibit a distinctive, sex-specific plasticity in dopamine transporter function and cocaine response after food restriction and exercise access; this plasticity is both absent in adults and not attributable to changes in dopamine transporter expression levels. These novel findings may help explain sex differences in vulnerability to eating disorders and drug abuse during adolescence.
This research was supported by the National Institutes of Health National Institute on Drug Abuse [Grant R21 DA038504 to L.C.D.], T.L.G. was supported by the NIH National Institute on Drug Abuse [Grant T32 DA031115 to Charles $P$. France]. Funding sources had no role in the generation, analyses, interpretations, or writing of these results. Interim results from this project have been previously published in abstract form in The FASEB Journal.

Previous presentations of works at meetings: Owens WA, Gilman TL, George CM, Metzel L, and Daws LC (2017) Impact of dietary restriction and exercise on dopamine transporter function in male and female adolescent and adult rats. The FASEB Journal 31(Suppl 1):987.14. Gilman TL, Owens WA, Metzel L, George CM, and Daws LC (2017) Sex-specific alterations in dopamine transporter function from food restriction and/or exercise are amplified during adolescence. Neuropsychopharmacology 43(Suppl 1): S476-S652 DOI: 10.1038/npp.2017.266. Gilman TL, Owens WA, George CM, Metzel L, and Daws LC (2018) Dopaminergic perturbations from food restriction and exercise are sex-dependently amplified during adolescence. The FASEB Journal 32(Suppl 1):682.6. Primary laboratory of origin: Daws.

https://doi.org/10.1124/jpet.119.260794.

S This article has supplemental material available at jpet.aspetjournals.org.

\section{Introduction}

Eating disorders afflict $\geq 3 \%$ of individuals in developed countries, and disproportionately impact females over males by more than double (Smink et al., 2014; Keski-Rahkonen and Mustelin, 2016). These disorders, which include anorexia nervosa (AN), bulimia nervosa, and binge-eating disorder, most often emerge during adolescence (Herpertz-Dahlmann, 2015). Indeed, adolescence is characterized not only by an increased risk of eating disorders, but also other neuropsychiatric disorders, as well as drug abuse and similar risky or compulsive behaviors (Andersen, 2003; Chambers et al., 2003; Paus et al., 2008; Arain et al., 2013; Holder and Blaustein, 2014).

Adolescence coincides with a particularly sensitive maturation period for the dopaminergic system (Chambers et al., 2003; Casey et al., 2008; Ernst et al., 2009; Novick et al., 2011; Sinclair et al., 2014; Fuhrmann et al., 2015; Niwa et al., 2016). Dopamine (DA) is a catecholamine neurotransmitter 
important for modulation of eating (Rolls et al., 1974; Yoshida et al., 1992; Volkow et al., 2003; Davis et al., 2009), motor activity (Andén et al., 1973; Baldo et al., 2002; Gallardo et al., 2014; Robertson et al., 2015; Felger and Treadway, 2017), emotion (Herman et al., 1982; Salgado-Pineda et al., 2005; Kienast et al., 2008; Belujon and Grace, 2015), impulsivity (van Gaalen et al., 2006; Buckholtz et al., 2010; Pine et al., 2010; Dalley and Robbins, 2017), and reward (Nestler and Carlezon, 2006; Johnson and Kenny, 2010; Eshel et al., 2016). Eating disorders, particularly AN and bulimia nervosa, are characterized by drastic changes in eating and/or activity behaviors that, when sufficiently rewarding to the individual's desired outcome (e.g., dramatic weight loss), can lead to compulsive engagement in such behavior despite severe deterioration in health or lifestyle. Consequently, DA pathophysiology is suspected to underlie, at least in part, adolescent-onset eating disorders (Barry and Klawans, 1976; Golden and Shenker, 1994; Frank et al., 2005; Frieling et al., 2010; Scherag et al., 2010; Kaye et al., 2013).

AN consistently has the highest mortality rate of all eating disorders (Arcelus et al., 2011; Preti et al., 2011; Keshaviah et al., 2014). Yet effective pharmacological treatments do not exist for any eating disorders. Antidepressant drugs, though beneficial for frequently comorbid mood disturbances, appear otherwise ineffective in treating $\mathrm{AN}$, and antipsychotic drugs have marginal if any effectiveness [see reviews by Carrera et al. (2014), Zipfel et al. (2015)]. One potential explanation for such weak treatment effectiveness is a lack of AN animal models. As with most neuropsychiatric disorders, modeling pathophysiology in animals such as rodents is inherently constrained by species differences and questions of emotional and intellectual complexity [refer to Gutierrez (2013), Bale et al. (2019)]. Nonetheless, introduction of specific disorder components, such as the varied physical and environmental stressors in the chronic mild stress model of depression (Willner, 2016), can be advantageous both for understanding neurophysiological disruptions (face validity) and for screening potential therapeutics (predictive validity). Here, we explored use of an adult activity-based anorexia (ABA) paradigm in early adolescence (postnatal day 30, P30) to examine how dopaminergic disruptions from food restriction and/or exercise might differ as a function of sex in adolescents and adults. Until now, animal investigations into anorexia have predominantly used adults, with the few examining adolescent animals missing the vulnerable early adolescent period (Kinzig and Hargrave, 2010; Aoki et al., 2012; BarbarichMarsteller et al., 2013a,b; Chowdhury et al., 2014; Frintrop et al., 2018a,b) during which these behaviors typically emerge in humans (approx. age 13 years) (Micali et al., 2014; Nagl et al., 2016).

The ABA paradigm is based upon work in the 1950s and 1960s that observed significantly enhanced running wheel activity in adult male rats under conditions of food restriction (Hall et al., 1953), to the extent that after approximately 2 weeks, animals ran themselves to death rather than eat when food was available (Routtenberg and Kuznesof, 1967). Current paradigm iterations closely resemble these original findings, involving unlimited running wheel access (exercise) plus duration-based (rather than quantity-based) food restriction (1 hour/day) (Carrera et al., 2014). To assess dopaminergic disruptions in the ABA paradigm, we measured DA transporter (DAT) function after food restriction, exercise, or their combination (ABA) in adult and adolescent rats of both sexes. Because DA uptake by DAT is a primary regulatory mechanism of dopamine signaling duration, measuring DAT function provides an excellent indication of dopaminergic tone (Cass and Gerhardt, 1994; Zahniser et al., 1999; Daws et al., 2002; Sabeti et al., 2003). To our knowledge, this is the first assessment of DAT function in an ABA paradigm.

\section{Materials and Methods}

Animals. Sprague-Dawley rats were bred in-house from breeders purchased through Taconic (NTac:SD; Rensselaer, NY), and all animals were maintained at $24^{\circ} \mathrm{C}$ on a $12: 12$ light/dark cycle, lights on at 0400 hours. Rats were weaned at P21, with P0 as day of birth. Rats were group-housed, two to three per cage, with samesex littermates on 7090 Teklad sani-chip bedding (Envigo, East Millstone, NJ) and provided ad libitum access to water and Teklad LM-485 mouse/rat sterilizable diet 7012 chow (Envigo) until commencement of experimental manipulations. All experiments were approved by the University of Texas Health Science Center at San Antonio Institutional Animal Care and Use Committee, and complied with the National Research Council's Guide for the Care and Use of Laboratory Animals, 8th Ed.

Activity-Based Anorexia Paradigm. Adolescent (P30 \pm 1 day) and adult (P90 \pm 5 days) male and female rats were randomly assigned to one of four treatment conditions: cage control (CC), exercise control (EC), food restricted control (FC), and ABA. Initial body weights were not different across treatment conditions within any age group (see Supplemental Table S1). Body weights, and food and water consumed, were measured once daily between 1500 and 1600 hours. Animals always began in the paradigm at 1600 hours; adults continued for 5 days [a standard adult paradigm lasts for between 8 and 14 days (Routtenberg and Kuznesof, 1967; Carrera et al., 2014)], but adolescents were restricted to 4 days due to survival issues during pilot chronoamperometry and cocaine-induced locomotion endpoints at 5 days. On the final day of their respective paradigm, rats were assigned to one of three endpoints (Fig. 1): blood and brain collection from drug-naive rats (i.e., not previously used in chronoamperometry or locomotor experiments) for blood hormone and quantitative autoradiography analyses; locomotor assay to measure acute, dose-dependent effects of cocaine, a DAT blocker; or in vivo high-speed chronoamperometry for measurement of DA uptake in the DAT-rich dorsal striatum, a brain region important for feeding behavior (Palmiter, 2008). Additional details are in the Supplemental Material.

Chemicals and Reagents. All chemicals and reagents were purchased from Sigma Aldrich (St. Louis, MO) unless otherwise indicated.

Locomotor Assay. Testing occurred between 1000 and 1500 hours on the final paradigm day. Locomotor activity was measured using beam breaks quantified in 1-minute bins with Multi-Varimex software (v2.10; Columbus Instruments, Columbus, OH). Animals first underwent a 45-minute habituation session; thereafter, rats received injections of vehicle $(0.9 \% \mathrm{NaCl}$, saline; $1 \mathrm{ml} / \mathrm{kg}$, i.p. $)$, then cocaine at increasing doses $(3.2,5.6$, and $10 \mathrm{mg} / \mathrm{kg})$ such that cumulative doses were $3.2,8.8$, and $18.8 \mathrm{mg} / \mathrm{kg}$, with locomotor activity recorded for 


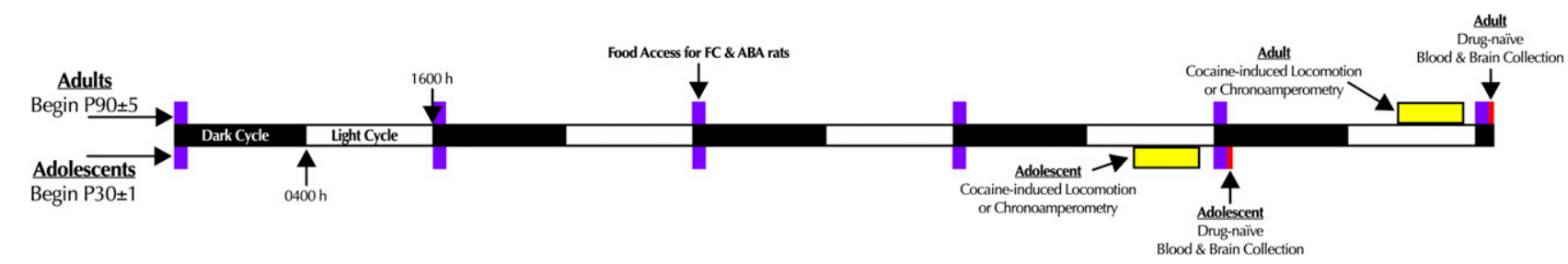

Fig. 1. Timeline of experiments. Adult male and female rats were singly housed in cages directly adjoining running wheels at postnatal day 90 (P90, \pm 5 days), whereas adolescent male and female rats were housed in the same setup at P30 ( \pm 1 day). Cage control (CC) and exercise control (EC) animals had 24-hour access to food, whereas food restricted control (FC) and activity-based anorexia (ABA) animals were given access to food for 1 hour per day (purple blocks) at the onset of the dark cycle (1600 hours, 12:12 dark/light cycle, lights on at 0400 hours). Adolescents went through the paradigm for 4 days, adults for 5 days. At the end of the paradigm, animals underwent one of three endpoints: chronoamperometry (between 0900 and 1500 hours; yellow block), cocaine-induced locomotion (between 1000 and 1500 hours; yellow block), or postprandial drug-naive blood and brain collection (1700 hours, red block).

15 minutes following each injection. For statistical comparisons, area under the curve (AUC) was calculated for the 15 minute period after saline and each cocaine injection using GraphPad Prism (v7.0e; GraphPad Software, La Jolla, CA). Additional details are in the Supplemental Material.

In Vivo High-Speed Chronoamperometry. Chronoamperometry involves measurement of the clearance of increasing amounts of exogenously applied DA from the extracellular space, at a time resolution of 100 milliseconds and spatial resolution of micrometers. This technique is an established tool for measuring DAT function in vivo and was performed as previously described (Baladi et al., 2015; Daws et al., 2016). Additional details are in the Supplemental Material.

[ ${ }^{125}$ I] RTI-55 Quantitative Autoradiography. Autoradiography was performed as previously detailed (Coulter et al., 1996). Additional details are in the Supplemental Material.

Statistical Analyses. Data were analyzed in each age group with a two-way (sex $\times$ condition) analysis of variance (ANOVA) in NCSS (v12.0.9; NCSS, LLC, Kaysville, UT) with Tukey-Kramer's post-hoc tests where appropriate to a priori compare across sex within condition, or across condition within sex. Statistical outcomes of three-way (day $\times$ sex $\times$ condition) repeated-measures ANOVA analyses of weights, with Geisser-Greenhouse correction for within-subjects analyses, are provided in Supplemental Table S2 for the drug-naive cohort of animals. Three-way (pmol of DA $\times$ sex $\times$ condition) repeated-measures ANOVA analyses of DA clearance rate, with Geisser-Greenhouse correction for within-subjects analyses, are provided in Supplemental Table S8. Data were graphed using GraphPad Prism (v7.0e; GraphPad Software). Significance was set a priori at $P<0.05$. Additional details are in the Supplemental Material.

\section{Results}

\section{Body Weights}

Initial weights were not different across treatment conditions in the drug-naive cohort, though expected sex differences in initial (day 0) weights were observed (Supplemental Table S1). By the end of the 4 or 5 day paradigm for adolescents and adults, respectively, significant weight differences across treatment conditions were noted. Day-by-day graphs of the time courses of body weights are provided in Supplemental Fig. S1, A-D, and three-way repeated-measure ANOVA outcomes relevant to these day-by-day measures are reported in Supplemental Table S2. Because all blood hormone, acute cocaine-induced locomotion, chronoamperometry, and autoradiography outcome measures took place on the final day of the paradigm, we focus here on final day weights, shown in Fig. 2.

Adolescents. As with day 0 weights, a significant main effect of sex was observed for day 4 body weights $[\mathrm{F}(1,68)=55.7$,
$P<0.001$ ), but unlike day 0 weights, a significant main effect of treatment condition was also detected $[\mathrm{F}(3,68)=69.3, P<$ 0.001]. No interactions between treatment and sex were detected. FC and ABA day 4 body weights were lower than both CC and EC groups within the same sex.

Adults. Though no significant sex $\times$ condition interaction was observed, significant main effects of $\operatorname{sex}(\mathrm{F}(1,57)=1039$, $P<0.001)$ and condition $(\mathrm{F}(3,57)=15.0, P<0.001)$ were found for day 5 body weights. Within adult females, only ABA animals had day 5 body weights different from CC animals. Weights of adult male ABA animals at day 5 were less than both CC and EC males.

\section{Food Consumption as Percent Body Weight}

Given the significant differences in body weights at the end of the paradigms for adolescent and adult rats in the drugnaive cohort, food consumption for each 24 hour period of the paradigm was calculated for each animal as a percentage of their previous day's body weight. Time-course graphs of these measures are presented in Supplemental Fig. S1, $\mathrm{E}-\mathrm{H}$, and as with body weights, we focus here on the final day of the adolescent (day 4) and adult (day 5) paradigms. Three-way repeated-measure ANOVA outcomes are reported in Supplemental Table S2.

Adolescents. A significant main effect of condition [(F3,68) $=222, P<0.001]$ was observed, with $\mathrm{FC}$ and $\mathrm{ABA}$ adolescents consuming less food than their EC and CC counterparts. No significant interaction between condition and sex, and no significant main effect of sex, were found. Adolescent EC males also consumed less food based on their body weight than CCs (Fig. 2C).

Adults. As with adolescents, condition displayed a significant main effect $[\mathrm{F}(3,57)=92.5, P<0.001]$. No significant main effect of sex was indicated, nor was a significant condition $\times$ sex interaction. Food intake was reduced in female FC and ABA conditions compared with female EC and CC conditions. Adult male body weight-based food intake on day 5 was reduced in EC, FC, and ABA animals relative to CC males. Male FC and ABA rats further exhibited reduced food intake relative to male EC animals (Fig. 2D).

\section{Running Wheel Activity}

Only animals in the EC and ABA conditions had unlocked running wheels (Fig. 3, A-D), so these two conditions were analyzed within age and across sex and condition. Area under the curve (AUC) was calculated for 23 hours during each paradigm day, and these AUC data per day are shown in 

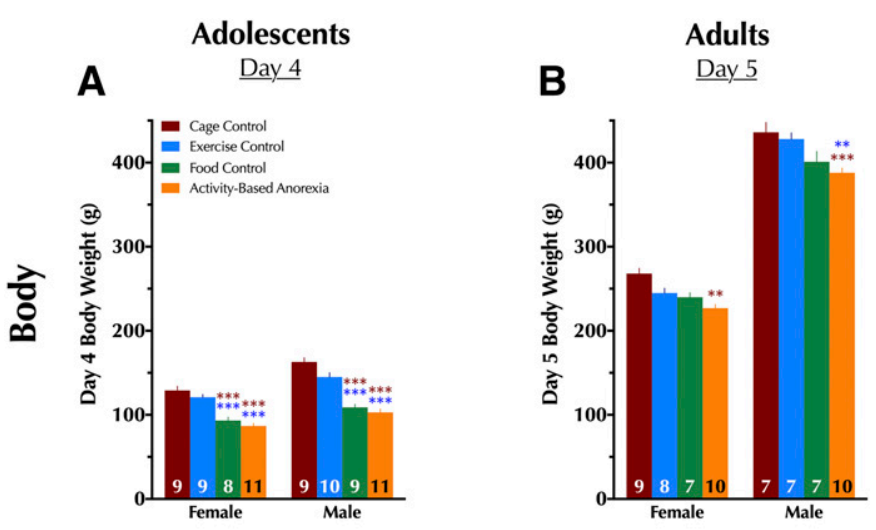

C

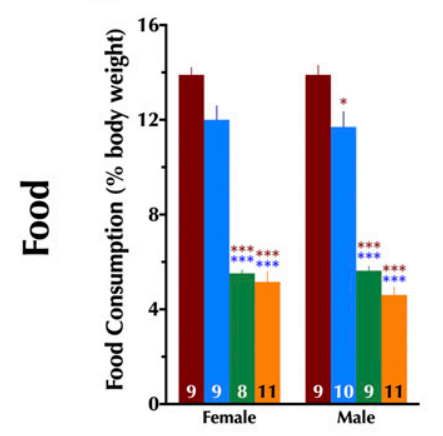

$\mathbf{E}$
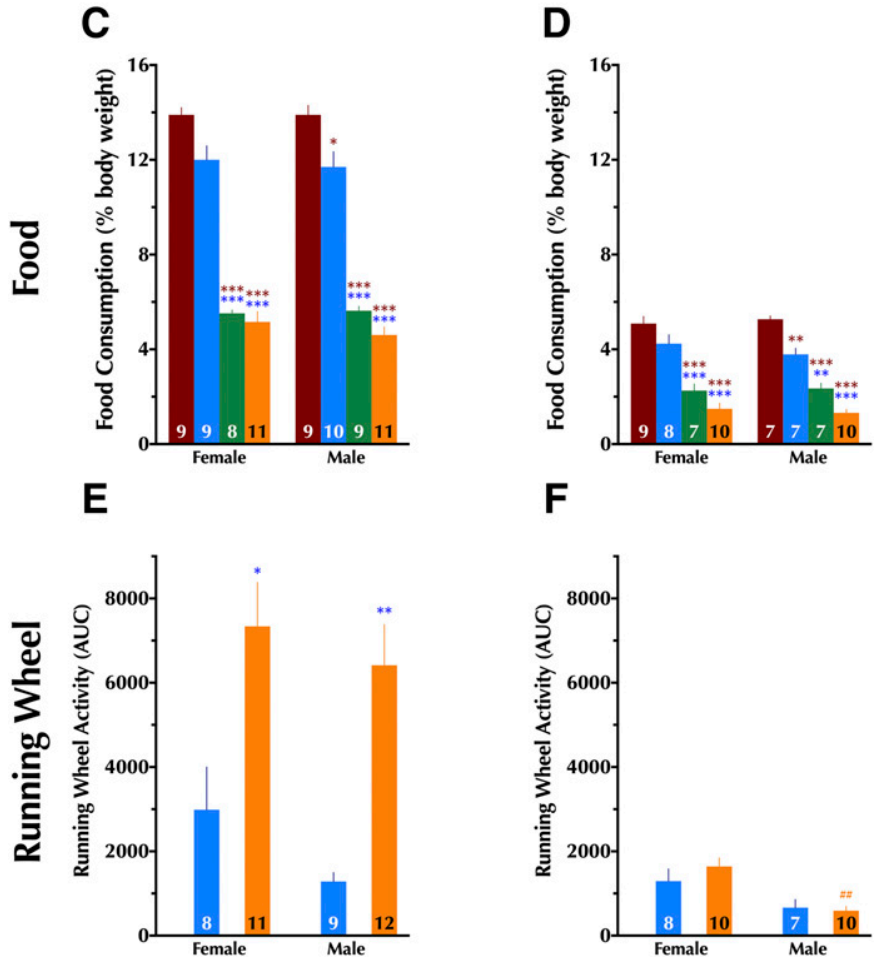$$
\mathbf{F}
$$

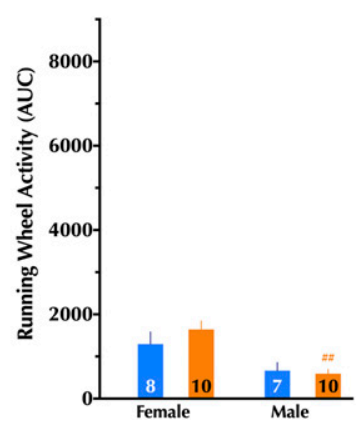

Fig. 2. Final day body weight, food consumption, and running wheel measures in drug-naive rats. The final day in the experimental paradigm for adolescents was day 4, whereas the final day for adults was day 5 . Because animals used for chronoamperometry and cocaineinduced locomotion were removed prior to completion of their full fourth (adolescents) or fifth (adults) day, only data from drug-naive rats are shown here. (A) Adolescent day 4 body weight; (B) adult day 5 body weight; (C) adolescent and (D) adult food consumption over the past 23 hours as a percentage of the individual animal's body weight; (E) adolescent and (F) adult running wheel activity over the last 23 hours for EC and ABA animals. No running wheel activity is shown for $\mathrm{CC}$ and FC animals because their wheels were locked and therefore they could not run in the wheel. Data were analyzed within each age group with a two-way (sex $\times$ condition) ANOVA with Tukey-Kramer's post-hoc tests where appropriate. Data are graphed as mean + S.E.M. $* P<0.05 ; * * P<0.01 ; * * * P<0.001$ vs. same-sex condition, indicated by color of asterisk(s), within the same age group. ${ }^{\# \#} P<0.01$ vs. same condition in females of same age group. Number of animals is indicated at base of each bar.

Fig. 3, E-H, with corresponding three-way repeated-measure ANOVA outcomes reported in Supplemental Table S3. Final paradigm day 4 AUCs for adolescents are in Fig. 2E, and final day 5 for adults are in Fig. 2 F.

Adolescents. A significant main effect of condition was detected $[\mathrm{F}(1,36)=25.9, P<0.001]$, with both female and male ABA animals exhibiting greater day 4 running wheel activity relative to their same-sex EC counterparts (Fig. 2E). Sex $\times$ condition interaction did not reach significance, nor did a main effect of sex.

Adults. There was no significant main effect of condition in adults, nor was there a significant sex $\times$ condition interaction. However, a main effect of sex was significant $[\mathrm{F}(1,31)=17.1$, $P<0.001$ ], with this driven by greater running wheel activity in female ABA animals compared with male ABA (Fig. 2F).

\section{Blood Hormone and Other Physiologic Measures}

See Supplemental Results and Supplemental Tables S4 and S5 for full details. Briefly, relative to same-sex CCs, circulating leptin and corticosterone were reduced in adolescent female ABA and FC groups, and adolescent female FCs also exhibited reduced ghrelin and increased insulin postprandial. Adolescent male blood hormone changes were similar for leptin and insulin, though no ghrelin or corticosterone differences relative to same-sex CCs were detected. In adults, only leptin levels were reduced compared to CCs in ABA animals (both sexes) and FCs (males). Brain water weights and plasma osmolality were unaffected in all groups. Hematocrit was elevated in adolescent ABAs (both sexes) compared to CCs, whereas only plasma protein was reduced selectively in adolescent female ABAs. No changes in either hematocrit or plasma protein were detected in any adults.

\section{Locomotor Activity}

AUC for locomotor activity, measured by minute-to-minute beam breaks, was calculated for the 15 minutes immediately following a saline injection, and for the combined three 15 minute periods immediately following three successive individual doses of cocaine $(3.2,5.6,10 \mathrm{mg} / \mathrm{kg})$. These AUCs were then analyzed to compare locomotor responses after injections.

Locomotor Activity after Saline. Saline AUCs are described in Supplemental Table S6, with corresponding time courses for saline and cocaine graphed in Fig. 4. For saline AUCs, no significant differences across treatment conditions were observed in either age group (Supplemental Table S6).

Locomotor Activity after Cocaine. Cocaine AUCs are inclusive of all three individual doses $(3.2,5.6,10 \mathrm{mg} / \mathrm{kg})$.

Adolescents. A significant sex $\times$ condition interaction was observed in the cocaine AUCs of adolescent rats $[\mathrm{F}(3,64)=5.11$, $P<0.01]$. Adolescent female ABA animals exhibited greater cocaine AUCs than EC counterparts (Fig. 4A). In contrast to adolescent females, adolescent males in the FC condition displayed greater cocaine AUCs than all other male conditions, and compared to female FC counterparts (Fig. 4B). Adolescent male ABA animals also exhibited higher cocaine-induced locomotion than male $\mathrm{CC}$ rats.

Adults. A main effect of sex was detected $[\mathrm{F}(1,66)=25.4$, $P<0.001$ ], with female CC rats displaying a higher cocaine AUC than male CCs (Fig. 4, C and D). Condition did not show a significant main effect, nor was there a significant sex $x$ condition interaction.

\section{In Vivo High-Speed Chronoamperometry}

For chronoamperometry, signal amplitudes at the highest amount of DA infused (100 pmol) were first evaluated to 
Adolescents
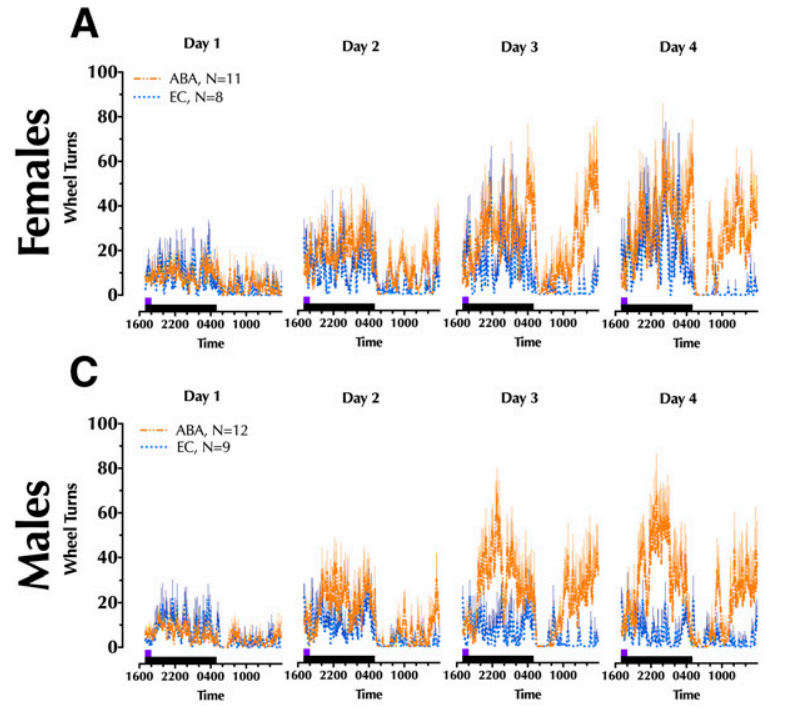

Adolescents

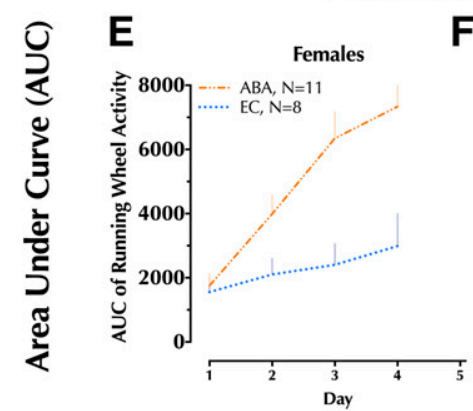

$\mathbf{F}$

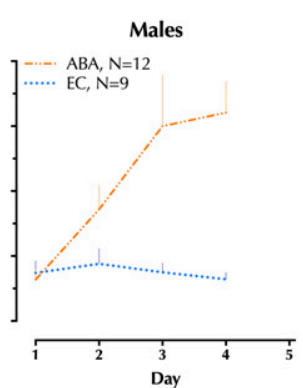

B
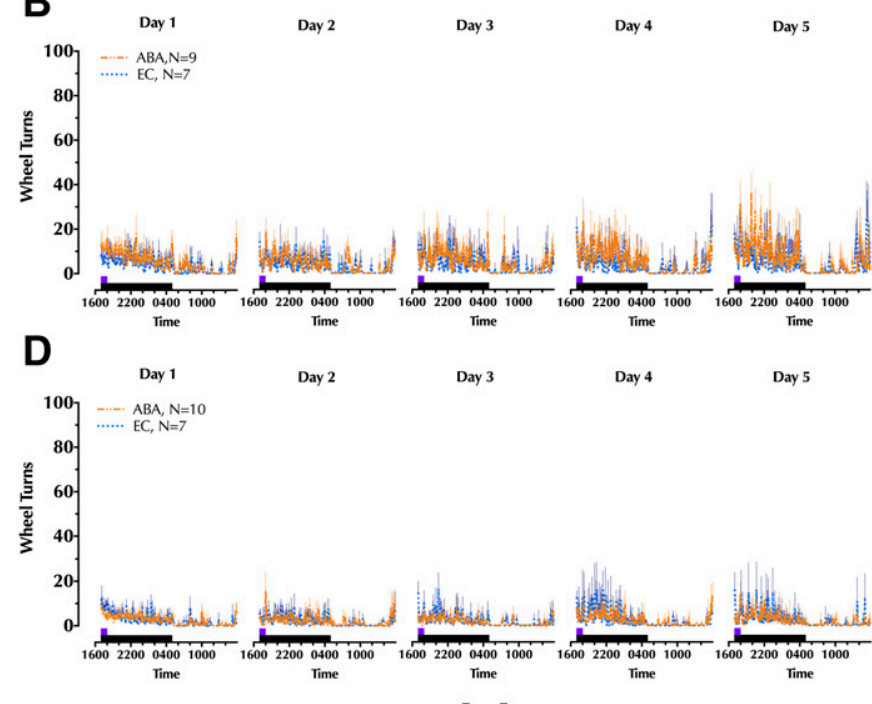

Day 2

Day 3

Day 4

Day 5

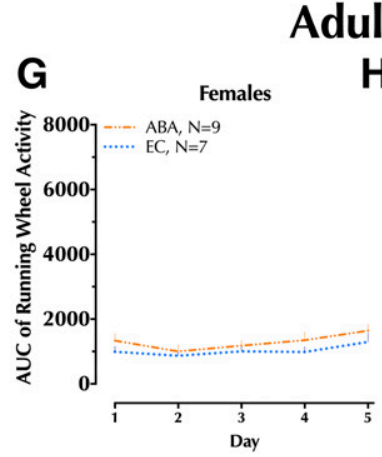

Fig. 3. Daily running wheel measures in drug-naive rats. Adolescents were run in the experimental paradigm for 4 days, whereas adults were run for 5 days. Because animals used for chronoamperometry and cocaine-induced locomotion were removed prior to completion of their full fourth (adolescents) or fifth (adults) day, only data from drug-naive rats are shown here. Collected in 5 minute bins, 23 hours of running wheel activity were recorded each day for (A) adolescent female and (B) male running wheel activity over four paradigm days; (C) adult female and (D) male running wheel activity over five paradigm days. Area under the curve (AUC) was calculated for each day from the graphs in (A-D), and are shown for (E) adolescent female and (F) male animals; (G) adult female and (H) male animals. No running wheel activity is shown for CC and FC animals, because their wheels were locked and therefore they could not run in the wheel. Only the final day of wheel running activity was analyzed (refer to Fig. 2, E and F). Data are graphed as mean + S.E.M. Number of animals is indicated in line legend: exercise control (EC), activity-based anorexia (ABA).

confirm that these were not significantly affected by treatment condition (Supplemental Table S7). The clearance rate of DA from the extracellular space was then plotted against picomoles of DA infused.

Adolescents. Significant interactions between sex $\times$ condition and condition $\times$ pmol of DA were observed (see Supplemental Table S8 for statistics). For comparisons across each treatment condition, a repeated-measures ANOVA was performed within each sex. In adolescent females, a significant main effect of condition was detected $[\mathrm{F}(3,185)=2.97, P<$ 0.05]. Specifically, at 40 and $50 \mathrm{pmol}$ of DA, female EC and FC rats had slower DA clearance relative to same-sex CCs (Fig. 5A). Clearance rates in female ABA rats were also greater at 40 and $50 \mathrm{pmol}$ of DA relative to $\mathrm{FC}$ rats. Adolescent males likewise exhibited a significant main effect of condition $[\mathrm{F}(3,155)=5.86, P<0.01]$, but in stark contrast to females, male ABA rats exhibited significantly impaired DA clearance relative to $\mathrm{CC}$ at DA levels of $20 \mathrm{pmol}$ and higher (Fig. 5B). As with females, male EC and FC rats exhibited significantly impaired DA clearance at 20,40, and $50 \mathrm{pmol}$, compared with male CCs.
Adults. Adult rats exhibited no significant interactions across sex, condition, and/or picomoles of DA (Supplemental Table S8; all $P>0.57$, though there was an expected significant main effect of picomoles of DA $[\mathrm{F}(5,235)=143, P<0.001]$ (Fig. 5, C and D).

\section{Adolescent DAT and Serotonin Transporter Expression}

Given that only adolescent rats exhibited functional changes in DAT as a result of condition, expression of DAT and serotonin transporter (SERT) was quantified using autoradiography in striatum and nucleus accumbens (NAc) of only adolescent animals. No significant differences in DAT or SERT expression were observed in either brain region in either sex, and no significant interactions between sex $\times$ condition were found (Fig. 6).

\section{Discussion}

Our key findings indicate that during adolescence, the dopaminergic system possesses a functional plasticity impacted not only by sex but also by dietary and behavioral patterns, such as 

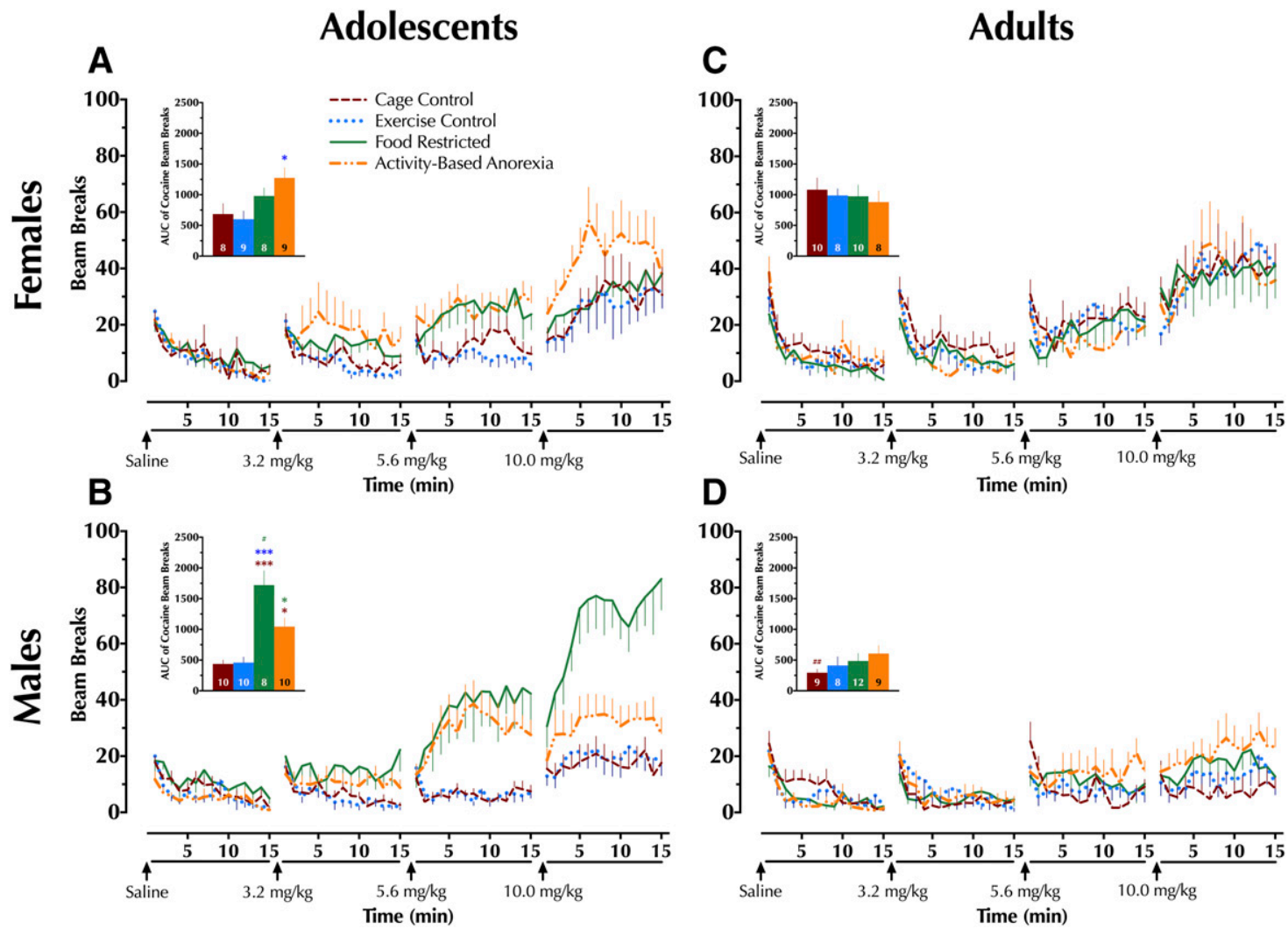

Fig. 4. Cocaine-induced locomotion. Rats were habituated to activity chambers for 45 minutes, then injected first with saline (1 ml/kg), then increasing individual doses of cocaine $(3.2,5.6$, and $10 \mathrm{mg} / \mathrm{kg})$ at 15 minute intervals in a cumulative-dosing paradigm. Activity was quantified as the number of beam breaks in 1 minute bins (lines in graphs), and the area under the curve (AUC) for all three cocaine doses is cumulatively shown in inset bar graphs for adolescent (A) female and (B) male and adult (C) female and (D) male rats. AUC data (inset bar graphs) were analyzed within each age group with a two-way (sex $\times$ condition) ANOVA with Tukey-Kramer's post-hoc tests where appropriate. Data are graphed as mean \pm S.E.M. $* P<0.05$; $* * * P<$ 0.001 vs. same-sex condition, indicated by color of asterisk(s), within the same age group. ${ }^{\#} P<0.05 ;{ }^{\# \#} P<0.01$ vs. same condition in females of same age group. Number of animals is indicated at base of each bar.

food restriction and exercise, in a manner not confounded by homeostatic body fluid disruptions or transporter expression changes. This responsive plasticity may feed forward to neurobehaviorally accelerate engagement in additional or continued unhealthy activities or compulsions, reshaping dopaminergic circuitry such that vulnerability to pervasive eating disorders or substance use disorders is perpetually augmented.

Being the first, to our knowledge, to apply the established adult ABA paradigm to early adolescence (P30), we observed striking vulnerability in adolescents compared with adults. Adult rats require at least 7 days to exhibit $\geq 20 \%$ starting body weight loss under these conditions (Routtenberg and Kuznesof, 1967); indeed, we observed minimal to no differences among treatment conditions in most adult endpoints. In contrast, adolescents responded so robustly that our experimentally planned 5-day paradigm was shortened to 4 days to facilitate survival for endpoint measures. This susceptibility of early adolescent rats parallels emergence in humans of pathologic behaviors and symptoms relating to eating disorders in early adolescence (age 13 years) (Micali et al., 2014; Nagl et al., 2016). The few studies using adolescent rats (all > P35) in an ABA paradigm have not validated the model (i.e., confirmed that key AN metabolic and hormonal manifestations are present), and seldom included all relevant controls, limiting interpretability of data (Kinzig and Hargrave, 2010; Aoki et al., 2012; Barbarich-Marsteller et al., 2013a,b; Chowdhury et al., 2014; Frintrop et al., 2018a,b). Moreover, the rapidity with which adolescent rats succumb to the adultbased ABA paradigm highlights the necessity for age-appropriate modifications that expand the experimental window beyond 4 days. Such a truncated window precludes the ability to investigate neurophysiological changes resulting from chronic ABA, and likewise hinders exploration of novel pharmacological interventions. Further, studies in adult rodents fail to reflect neural mechanisms active in the most vulnerable population, adolescents, during a time when brain maturation occurs. Considering AN's high mortality (Arcelus et al., 2011; Preti et al., 2011; Keshaviah et al., 2014), as well as its long-term stability in those who survive (Nagl et al., 2016), a chronic ABA paradigm commencing during the vulnerable early adolescent period could provide a much-needed model in AN pathophysiology and treatment studies.

Blood hormone measurements further evidence the need for an optimized early adolescent ABA model. AN is characterized by substantial baseline reductions in circulating insulin and leptin, along with increases in ghrelin and cortisol (Monteleone et al., 2000; Nakahara et al., 2008; KarczewskaKupczewska et al., 2010; Korek et al., 2013). With the exception of reduced leptin levels, none of these hormonal disruptions were mirrored in adolescents, underscoring the poor face validity of the current adult-based paradigm in adolescents. Recent efforts have prolonged survival of adolescent female rats in a modified ABA protocol (Frintrop et al., 2018b), but it remains to be seen whether this procedure elicits any 

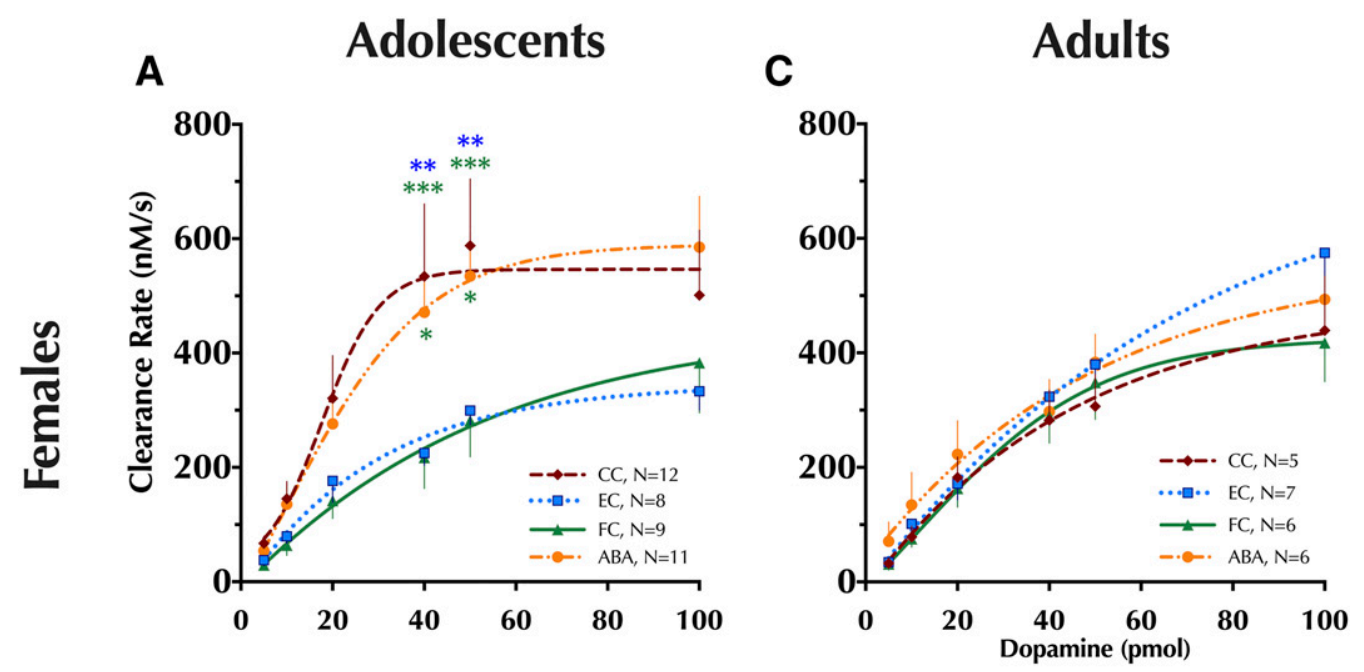

B

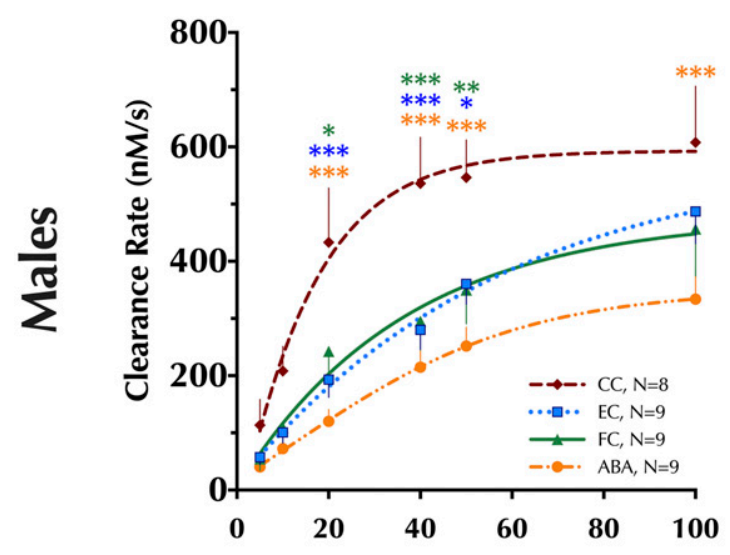

D

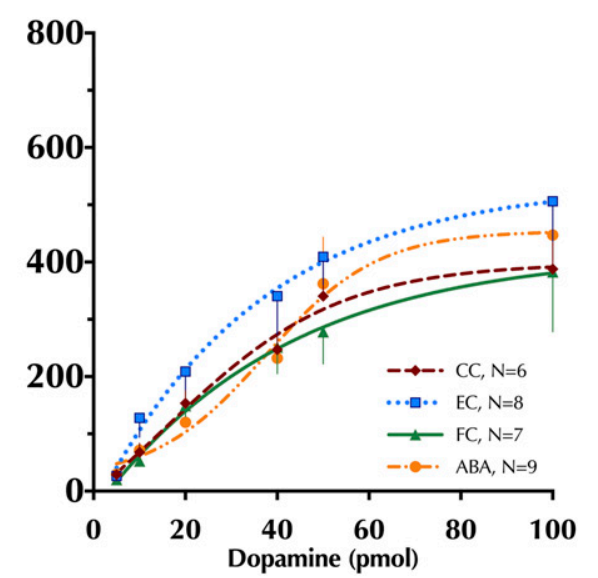

Fig. 5. Striatal dopamine clearance measured using in vivo high-speed chronoamperometry. Clearance rate of dopamine in the striatum of adolescent (A) female and (B) male rats and adult (C) female and (D) male rats is shown for increasing amounts of exogenously infused dopamine. Four-parameter logistic regressions with baseline constrained at 0 were used to generate curve fits. Data analyzed within each age and sex group using a repeatedmeasures (pmol DA $\times$ condition) ANOVA with Tukey-Kramer's post-hoc tests where appropriate. Data are graphed as mean \pm S.E.M. $* P<0.05$; $* * P<$ $0.01 ; * * P<0.001$ vs. same-sex condition, indicated by color of asterisk(s), within the same picomole amount. Number of animals is indicated in respective legends: cage control (CC), exercise control (EC), food restricted control (FC), activity-based anorexia (ABA).

sex-dependent effects, or reflects any hallmark blood hormone disruptions observed in AN patients. Another alternative approach to modeling AN uses a behavioral economics framework (Rowland et al., 2018), though how well this can be adapted to adolescents is undetermined.

Homeostatic body fluid maintenance, as evidenced by plasma osmolality and brain water weight, was not disrupted in FC and ABA adolescents, despite dramatic reductions in food and water consumption. Importantly, this suggests that the observed plasticity in DAT function is not confounded by dehydration, differences in DA diffusion, or changes in tortuosity at the carbon fiber microelectrode site.

The elevated hematocrit observed in adolescent $\mathrm{ABA}$ rats of both sexes, and adolescent male FC rats, is at odds with reports of reduced hematocrit levels in food restricted rats (Wojciak, 2014) and patients with severe AN [but see Symreng et al. (1985), Sabel et al. (2013)]. Instead, increased hematocrit is usually observed after excessive exercise in humans (Skarpańska-Stejnborn et al., 2015) but not rodents (Tian et al., 2012; Tang et al., 2019). Plasma protein levels were diminished only in adolescent female ABA rats, which could be attributable to muscle wasting or liver inflammation, both commonly observed in patients with AN (Rosen et al., 2017).

Despite the limitations of applying the adult ABA paradigm to adolescents, the current investigation revealed striking age- and sex-specific effects of exercise access plus food restriction on the locomotor-stimulating effects of the drug of abuse, cocaine. Cocaine-mediated blockade of DA uptake through DAT corresponds to cocaine-induced locomotor responses in Sprague-Dawley rats (Sabeti et al., 2002; Gulley et al., 2003). Thus, this behavior assay can serve as a proxy, at least in part, to indicate individual differences in DAT function as revealed by cocaine blockade.

To our knowledge, the influence of food restriction alone on adolescent or female locomotor activity in response to cocaine had not previously been explored, though the observed augmentation of cocaine response in adolescent males fits with previous, more extended periods of food restriction (1-2 weeks) in adult males (Bell et al., 1997). The absence of changes here in cocaine locomotor response after exercise alone across age and sex indicate that its effects take more than 4-5 days to emerge behaviorally, in agreement with multiple reports (Cosgrove et al., 2002; Smith et al., 2008a,b; 

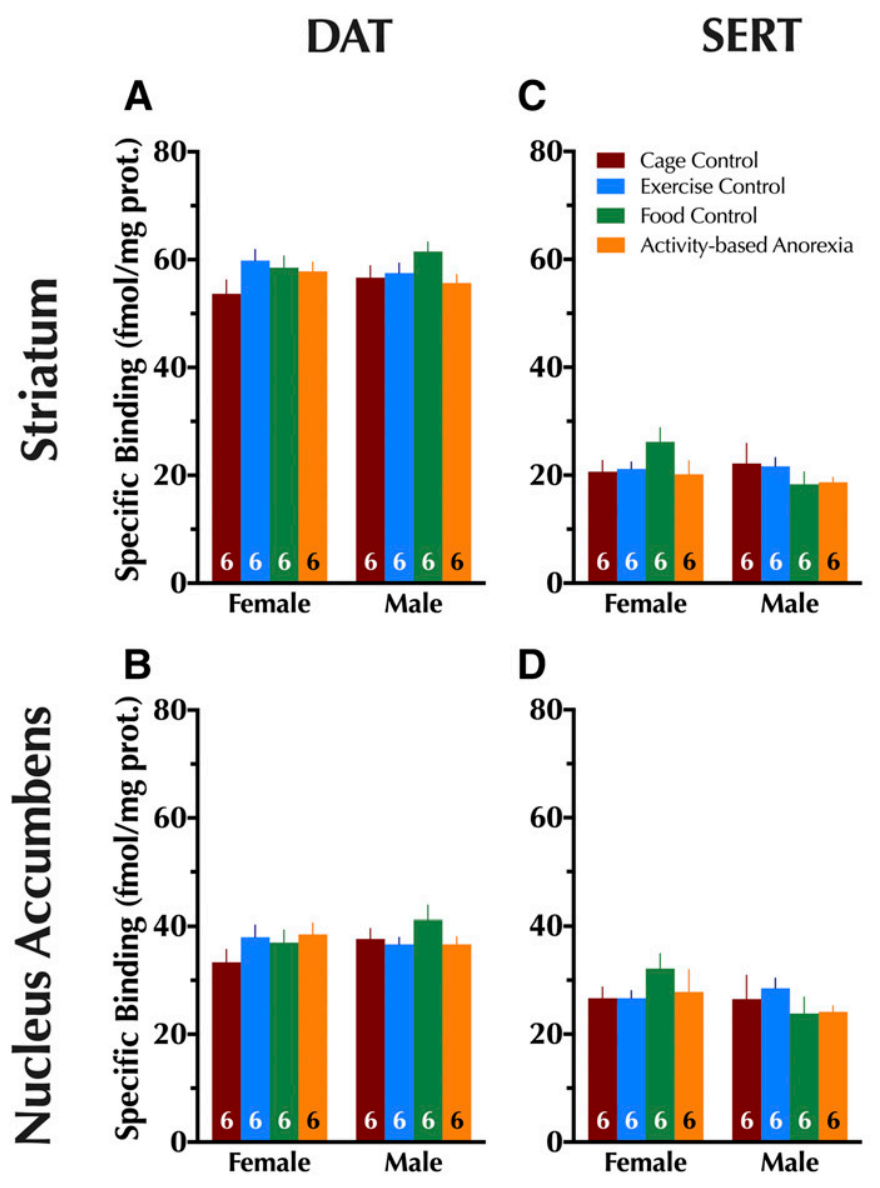

D

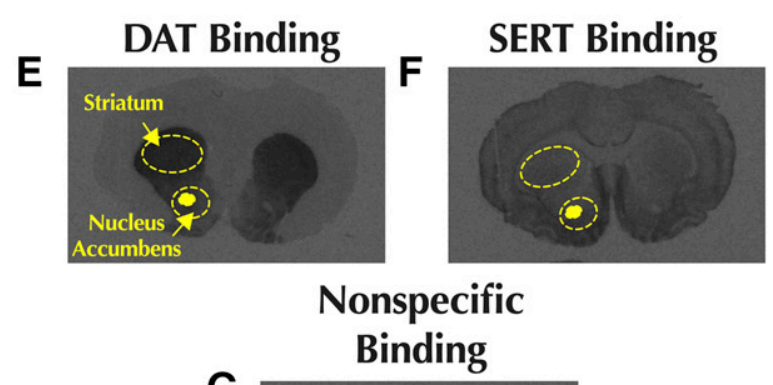

G

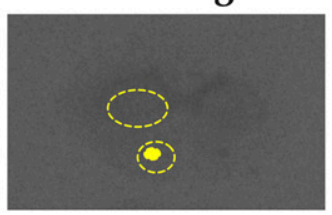

Fig. 6. Quantitative autoradiography in adolescents for dopamine and serotonin transporter expression. Specific binding for dopamine transporter is shown in adolescent (A) striatum and (B) nucleus accumbens. Specific binding for serotonin transporter is also shown in adolescent (C) striatum and (D) nucleus accumbens. Representative brain sections illustrating (E) DAT, (F) SERT, and (G) nonspecific binding are shown with the analyzed regions for striatum and nucleus accumbens illustrated with dashed circles. Data were analyzed within each age group with a twoway (sex $\times$ condition) ANOVA. Data are graphed as mean + S.E.M. Number of animals is indicated at base of each bar.

Smith et al., 2011; Smith and Witte, 2012; Zlebnik et al., 2012). Most striking is that the combination of exercise plus food restriction in adolescent males substantially attenuated the locomotor response to cocaine, relative to FCs, but that this combination produced the largest behavioral response to cocaine in adolescent females. This is in accord with a disproportionately high prevalence of eating disorder diagnoses, particularly of $\mathrm{AN}$, among females with comorbid substance use disorders [see Harrop and Marlatt (2010) for review]. The seemingly augmented response of ABA adolescent females to cocaine, versus the moderated response in ABA adolescent males, likely reflects underlying sex- and age-dependent plasticity in DAT function in response to the combination of food restriction and exercise.

Indeed, we directly investigated DAT function in dorsal striatum, where DAT expression is high, and DA signaling influences reward, activity, and eating behaviors (Sotak et al., 2005; Palmiter, 2007, 2008; Tomasi and Volkow, 2013). To our knowledge, this is the first characterization of in vivo DAT function in adolescent females, and the first evaluation of DAT functional changes after an ABA paradigm. We found a robust age difference in DA uptake across sexes, with faster clearance in adolescents compared with adults. Moreover, only in adolescents did ABA reveal a sex-specific effect, with dramatic functional DAT reductions observed in males but not females. Reductions in adolescent DAT function in ECs and FCs support the observed slowing of DA clearance in adolescent male ABAs, making the lack of attenuated DAT function in adolescent ABA females remarkable. Under prolonged ( $>4$ days) ABA conditions, DAT function in adolescent females might eventually either increase, or persist at an adolescent CC-like level, reflecting clinical reports of significant DAT upregulation in female patients suffering from AN for an average of 10 years (Frieling et al., 2010). Optimization of the ABA paradigm for adolescents, to enable prolonged study with more of the characteristic hormonal disruptions in place, will permit study of this possibility. Importantly, the functional changes in DAT are not mirrored by any significant changes in total striatal DAT or SERT expression, the latter of which can transport DA under conditions of impaired DAT function (Larsen et al., 2011). These results suggest that DA clearance rate shifts are probably the result of changes in intrinsic activity and/or plasma membrane expression of DAT.

The sex-specific adolescent plasticity in dorsal striatum DAT function does not directly map on to cocaine-induced locomotor activity. This could be attributable to brain region-specific effects of cocaine on DA clearance, as cocaine-mediated blockade of DAT in NAc corresponds more closely to druginduced changes in locomotor activity, compared to dorsal striatum (Sabeti et al., 2002). However, this is not attributable to any detectable changes in DAT expression or cocaine affinity in NAc (Gulley et al., 2003); indeed, we observed no significant differences in NAc DAT expression here. Thus, different cocaine-induced locomotor responses across conditions in female and male adolescents might reflect functional NAc DAT differences, whereas the functional changes measured using chronoamperometry in dorsal striatum could indicate DA clearance shifts that impact more habitual behaviors (e.g., home cage or running wheel activity).

Certainly, this plasticity could instead be secondary to other neurophysiological disruptions in the dopaminergic system (e.g., changes in DA release) or other neurotransmitter systems (Hillebrand et al., 2005; Atchley and Eckel, 2006), and could be influenced by apparent circadian changes in the adolescent ABA groups during the final 2 days of the paradigm (see Fig. 3). Nonetheless, the present findings suggest that striatal DAT function should be a focus of future studies into the mechanisms underlying eating disorder and drug abuse 
vulnerability. Moreover, a chronic ABA paradigm commencing during the vulnerable early adolescent period would provide a much-needed model to study AN pathophysiology and treatment.

\section{Acknowledgments}

We thank Dr. Charles P. France for the generous use of his rat locomotor chambers, Dr. Wouter Koek for his expert assistance with statistical analyses, and Dr. Rheaclare Fraser-Spears for assistance with tissue collection.

\section{Authorship Contributions}

Participated in research design: Gilman, George, Toney, Daws.

Conducted experiments: Gilman, Owens, George, Metzel, Vitela,

Ferreira, Bowman, Gould.

Performed data analysis: Gilman, Daws.

Wrote or contributed to the writing of the manuscript: Gilman, Gould, Toney, Daws.

\section{References}

Andén NE, Strömbom U, and Svensson TH (1973) Dopamine and noradrenaline receptor stimulation: reversal of reserpine-induced suppression of motor activity. Psychopharmacology (Berl) 29:289-298.

Andersen SL (2003) Trajectories of brain development: point of vulnerability or window of opportunity? Neurosci Biobehav Rev 27:3-18.

Aoki C, Sabaliauskas N, Chowdhury T, Min J-Y, Colacino AR, Laurino K, and Barbarich-Marsteller NC (2012) Adolescent female rats exhibiting activitybased anorexia express elevated levels of GABA(A) receptor $\alpha 4$ and $\delta$ subunits at the plasma membrane of hippocampal CA1 spines. Synapse 66:391-407.

Arain M, Haque M, Johal L, Mathur P, Nel W, Rais A, Sandhu R, and Sharma S (2013) Maturation of the adolescent brain. Neuropsychiatr Dis Treat 9:449-461.

Arcelus J, Mitchell AJ, Wales J, and Nielsen S (2011) Mortality rates in patients with anorexia nervosa and other eating disorders. A meta-analysis of 36 studies. Arch Gen Psychiatry 68:724-731.

Atchley DP and Eckel LA (2006) Treatment with 8-OH-DPAT attenuates the weight loss associated with activity-based anorexia in female rats. Pharmacol Biochem Behav 83:547-553.

Baladi MG, Horton RE, Owens WA, Daws LC, and France CP (2015) Eating high fat chow decreases dopamine clearance in adolescent and adult male rats but selectively enhances the locomotor stimulating effects of cocaine in adolescents Int $J$ Neuropsychopharmacol 18:pyv024.

Baldo BA, Sadeghian K, Basso AM, and Kelley AE (2002) Effects of selective dopamine D1 or D2 receptor blockade within nucleus accumbens subregions on ingestive behavior and associated motor activity. Behav Brain Res 137:165-177.

Bale TL, Abel T, Akil H, Carlezon WA Jr, Moghaddam B, Nestler EJ, Ressler KJ, and Thompson SM (2019) The critical importance of basic animal research for neuropsychiatric disorders. Neuropsychopharmacology 44:1349-1353.

Barbarich-Marsteller NC, Fornal CA, Takase LF, Bocarsly ME, Arner C, Walsh BT, Hoebel BG, and Jacobs BL (2013a) Activity-based anorexia is associated with reduced hippocampal cell proliferation in adolescent female rats. Behav Brain Res 236:251-257.

Barbarich-Marsteller NC, Underwood MD, Foltin RW, Myers MM, Walsh BT, Barrett JS, and Marsteller DA (2013b) Identifying novel phenotypes of vulnerability and resistance to activity-based anorexia in adolescent female rats Int J Eat Disord 46:737-746.

Barry VC and Klawans HL (1976) On the role of dopamine in the pathophysiology of anorexia nervosa. J Neural Transm (Vienna) 38:107-122.

Bell SM, Stewart RB, Thompson SC, and Meisch RA (1997) Food-deprivation increases cocaine-induced conditioned place preference and locomotor activity in rats. Psychopharmacology (Berl) 131:1-8.

Belujon P and Grace AA (2015) Regulation of dopamine system responsivity and its adaptive and pathological response to stress. Proc Biol Sci 282:20142516.

Buckholtz JW, Treadway MT, Cowan RL, Woodward ND, Li R, Ansari MS, Baldwin RM, Schwartzman AN, Shelby ES, Smith CE, et al. (2010) Dopaminergic network differences in human impulsivity. Science 329:532.

Carrera O, Fraga Á, Pellón R, and Gutiérrez E (2014) Rodent model of activity-based anorexia, Curr Protoc Neurosci 67, p 9.47.1-9.47.11.

Casey BJ, Jones RM, and Hare TA (2008) The adolescent brain. Ann N Y Acad Sci 1124:111-126.

Cass WA and Gerhardt GA (1994) Direct in vivo evidence that D2 dopamine receptors can modulate dopamine uptake. Neurosci Lett 176:259-263.

Chambers RA, Taylor JR, and Potenza MN (2003) Developmental neurocircuitry of motivation in adolescence: a critical period of addiction vulnerability. Am J Psychiatry 160:1041-1052.

Chowdhury TG, Ríos MB, Chan TE, Cassataro DS, Barbarich-Marsteller NC, and Aoki C (2014) Activity-based anorexia during adolescence disrupts normal development of the CA1 pyramidal cells in the ventral hippocampus of female rats. Hippocampus 24:1421-1429.

Cosgrove KP, Hunter RG, and Carroll ME (2002) Wheel-running attenuates intravenous cocaine self-administration in rats: sex differences. Pharmacol Biochem Behav 73:663-671.

Coulter CL, Happe HK, and Murrin LC (1996) Postnatal development of the dopamine transporter: a quantitative autoradiographic study. Brain Res Dev Brain Res 92:172-181.
Dalley JW and Robbins TW (2017) Fractionating impulsivity: neuropsychiatric implications. Nat Rev Neurosci 18:158-171.

Davis CA, Levitan RD, Reid C, Carter JC, Kaplan AS, Patte KA, King N, Curtis C, and Kennedy JL (2009) Dopamine for "wanting" and opioids for "liking": a comparison of obese adults with and without binge eating. Obesity (Silver Spring) 17: $1220-1225$

Daws LC, Callaghan PD, Morón JA, Kahlig KM, Shippenberg TS, Javitch JA, and Galli A (2002) Cocaine increases dopamine uptake and cell surface expression of dopamine transporters. Biochem Biophys Res Commun 290:1545-1550.

Daws LC, Owens WA, and Toney GM (2016) Using High-Speed Chronoamperometry to Measure Biogenic Amine Release and Uptake In Vivo, in Neurotransmitter Transporters (Bönisch H and Sitte H eds) 118, pp 53-81, Humana Press, New York.

Ernst M, Romeo RD, and Andersen SL (2009) Neurobiology of the development of motivated behaviors in adolescence: a window into a neural systems model. Pharmacol Biochem Behav 93:199-211.

Eshel N, Tian J, Bukwich M, and Uchida N (2016) Dopamine neurons share common response function for reward prediction error. Nat Neurosci 19:479-486.

Felger JC and Treadway MT (2017) Inflammation effects on motivation and motor activity: role of dopamine. Neuropsychopharmacology 42:216-241.

Frank GK, Bailer UF, Henry SE, Drevets W, Meltzer CC, Price JC, Mathis CA, Wagner A, Hoge J, Ziolko S, et al. (2005) Increased dopamine D2/D3 receptor binding after recovery from anorexia nervosa measured by positron emission tomography and [11c]raclopride. Biol Psychiatry 58:908-912.

Frieling H, Römer KD, Scholz S, Mittelbach F, Wilhelm J, De Zwaan M, Jacoby GE, Kornhuber J, Hillemacher T, and Bleich S (2010) Epigenetic dysregulation of dopaminergic genes in eating disorders. Int J Eat Disord 43:577-583.

Frintrop L, Liesbrock J, Paulukat L, Johann S, Kas MJ, Tolba R, Heussen N, Neulen J, Konrad K, Herpertz-Dahlmann B, et al. (2018a) Reduced astrocyte density underlying brain volume reduction in activity-based anorexia rats. World $J$ Biol Psychiatry 19:225-235.

Frintrop L, Trinh S, Liesbrock J, Paulukat L, Kas MJ, Tolba R, Konrad K, HerpertzDahlmann B, Beyer C, and Seitz J (2018b) Establishment of a chronic activitybased anorexia rat model. J Neurosci Methods 293:191-198.

Fuhrmann D, Knoll LJ, and Blakemore S-J (2015) Adolescence as a sensitive period of brain development. Trends Cogn Sci 19:558-566.

Gallardo CM, Darvas M, Oviatt M, Chang CH, Michalik M, Huddy TF, Meyer EE, Shuster SA, Aguayo A, Hill EM, et al. (2014) Dopamine receptor 1 neurons in the dorsal striatum regulate food anticipatory circadian activity rhythms in mice. eLife 3:e03781.

Golden NH and Shenker IR (1994) Amenorrhea in anorexia nervosa. Neuroendocrine control of hypothalamic dysfunction. Int J Eat Disord 16:53-60.

Gulley JM, Hoover BR, Larson GA, and Zahniser NR (2003) Individual differences in cocaine-induced locomotor activity in rats: behavioral characteristics, cocaine pharmacokinetics, and the dopamine transporter. Neuropsychopharmacology 28:2089-2101.

Gutierrez E (2013) A rat in the labyrinth of anorexia nervosa: contributions of the activity-based anorexia rodent model to the understanding of anorexia nervosa. Int $J$ Eat Disord 46:289-301.

Hall JF, Smith K, Schnitzer SB, and Hanford PV (1953) Elevation of activity level in the rat following transition from ad libitum to restricted feeding. J Comp Physiol Psychol 46:429-433.

Harrop EN and Marlatt GA (2010) The comorbidity of substance use disorders and eating disorders in women: prevalence, etiology, and treatment. Addict Behav 35:392-398.

Herman JP, Guillonneau D, Dantzer R, Scatton B, Semerdjian-Rouquier L, and Le Moal M (1982) Differential effects of inescapable footshocks and of stimuli previously paired with inescapable footshocks on dopamine turnover in cortical and limbic areas of the rat. Life Sci 30:2207-2214.

Herpertz-Dahlmann B (2015) Adolescent eating disorders: update on definitions, symptomatology, epidemiology, and comorbidity. Child Adolesc Psychiatr Clin N Am 24:177-196.

Hillebrand JJ, van Elburg AA, Kas MJ, van Engeland H, and Adan RA (2005) Olanzapine reduces physical activity in rats exposed to activity-based anorexia: possible implications for treatment of anorexia nervosa? Biol Psychiatry 58:651-657.

Holder MK and Blaustein JD (2014) Puberty and adolescence as a time of vulnerability to stressors that alter neurobehavioral processes. Front Neuroendocrinol 35:89-110.

Johnson PM and Kenny PJ (2010) Dopamine D2 receptors in addiction-like reward dysfunction and compulsive eating in obese rats [published correction appears in Nat Neurosci (2010) 13:1033]. Nat Neurosci 13:635-641.

Karczewska-Kupczewska M, Strączkowski M, Adamska A, Nikołajuk A, Otziomek E Górska M, and Kowalska I (2010) Increased suppression of serum ghrelin concentration by hyperinsulinemia in women with anorexia nervosa. Eur J Endocrinol 162:235-239.

Kaye WH, Wierenga CE, Bailer UF, Simmons AN, and Bischoff-Grethe A (2013) Nothing tastes as good as skinny feels: the neurobiology of anorexia nervosa. Trends Neurosci 36:110-120.

Keshaviah A, Edkins K, Hastings ER, Krishna M, Franko DL, Herzog DB, Thomas JJ, Murray HB, and Eddy KT (2014) Re-examining premature mortality in anorexia nervosa: a meta-analysis redux. Compr Psychiatry 55:1773-1784. Keski-Rahkonen A and Mustelin L (2016) Epidemiology of eating disorders in Europe: prevalence, incidence, comorbidity, course, consequences, and risk factors. Curr Opin Psychiatry 29:340-345.

Kienast T, Hariri AR, Schlagenhauf F, Wrase J, Sterzer P, Buchholz HG, Smolka MN, Gründer G, Cumming P, Kumakura Y, et al. (2008) Dopamine in amygdala gates limbic processing of aversive stimuli in humans. Nat Neurosci 11:1381-1382.

Kinzig KP and Hargrave SL (2010) Adolescent activity-based anorexia increases anxiety-like behavior in adulthood. Physiol Behav 101:269-276. 
Korek E, Krauss H, Gibas-Dorna M, Kupsz J, Piątek M, and Piątek J (2013) Fasting and postprandial levels of ghrelin, leptin and insulin in lean, obese and anorexic subjects. Prz Gastroenterol 8:383-389.

Larsen MB, Sonders MS, Mortensen OV, Larson GA, Zahniser NR, and Amara SG (2011) Dopamine transport by the serotonin transporter: a mechanistically distinct mode of substrate translocation. J Neurosci 31:6605-6615.

Micali N, Ploubidis G, De Stavola B, Simonoff E, and Treasure J (2014) Frequency and patterns of eating disorder symptoms in early adolescence. $J$ Adolesc Health 54:574-581.

Monteleone P, Di Lieto A, Tortorella A, Longobardi N, and Maj M (2000) Circulating leptin in patients with anorexia nervosa, bulimia nervosa or binge-eating disorder: relationship to body weight, eating patterns, psychopathology and endocrine changes. Psychiatry Res 94:121-129.

Nagl M, Jacobi C, Paul M, Beesdo-Baum K, Höfler M, Lieb R, and Wittchen H-U (2016) Prevalence, incidence, and natural course of anorexia and bulimia nervosa among adolescents and young adults. Eur Child Adolesc Psychiatry 25:903-918.

Nakahara T, Harada T, Yasuhara D, Shimada N, Amitani H, Sakoguchi T, Kamiji MM, Asakawa A, and Inui A (2008) Plasma obestatin concentrations are negatively correlated with body mass index, insulin resistance index, and plasma leptin concentrations in obesity and anorexia nervosa. Biol Psychiatry 64:252-255.

Nestler EJ and Carlezon WA Jr (2006) The mesolimbic dopamine reward circuit in depression. Biol Psychiatry 59:1151-1159.

Niwa M, Lee RS, Tanaka T, Okada K, Kano S, and Sawa A (2016) A critical period of vulnerability to adolescent stress: epigenetic mediators in mesocortical dopaminergic neurons. Hum Mol Genet 25:1370-1381.

Novick AM, Forster GL, Tejani-Butt SM, and Watt MJ (2011) Adolescent social defeat alters markers of adult dopaminergic function. Brain Res Bull 86:123-128.

Palmiter RD (2007) Is dopamine a physiologically relevant mediator of feeding behavior? Trends Neurosci 30:375-381.

Palmiter RD (2008) Dopamine signaling in the dorsal striatum is essential for motivated behaviors: lessons from dopamine-deficient mice. Ann N Y Acad Sci 1129:35-46.

Paus T, Keshavan M, and Giedd JN (2008) Why do many psychiatric disorders emerge during adolescence? Nat Rev Neurosci 9:947-957.

Pine A, Shiner T, Seymour B, and Dolan RJ (2010) Dopamine, time, and impulsivity in humans. J Neurosci 30:8888-8896.

Preti A, Rocchi MB, Sisti D, Camboni MV, and Miotto P (2011) A comprehensive metaanalysis of the risk of suicide in eating disorders. Acta Psychiatr Scand 124:6-17.

Robertson CL, Ishibashi K, Mandelkern MA, Brown AK, Ghahremani DG, Sabb F, Bilder R, Cannon T, Borg J, and London ED (2015) Striatal D1- and D2-type dopamine receptors are linked to motor response inhibition in human subjects. J Neurosci 35:5990-5997.

Rolls ET, Rolls BJ, Kelly PH, Shaw SG, Wood RJ, and Dale R (1974) The relative attenuation of self-stimulation, eating and drinking produced by dopaminereceptor blockade. Psychopharmacology (Berl) 38:219-230.

Rosen E, Bakshi N, Watters A, Rosen HR, and Mehler PS (2017) Hepatic complications of anorexia nervosa. Dig Dis Sci 62:2977-2981.

Routtenberg A and Kuznesof AW (1967) Self-starvation of rats living in activity wheels on a restricted feeding schedule. J Comp Physiol Psychol 64:414-421.

Rowland NE, Atalayer D, Cervantez MR, Minaya DM, and Splane EC (2018) Costbased anorexia: a novel framework to model anorexia nervosa. Appetite 130:50-58.

Sabel AL, Gaudiani JL, Statland B, and Mehler PS (2013) Hematological abnormalities in severe anorexia nervosa. Ann Hematol 92:605-613.

Sabeti J, Gerhardt GA, and Zahniser NR (2002) Acute cocaine differentially alters accumbens and striatal dopamine clearance in low and high cocaine locomotor responders: behavioral and electrochemical recordings in freely moving rats. $J$ Pharmacol Exp Ther 302:1201-1211.

Sabeti J, Gerhardt GA, and Zahniser NR (2003) Chloral hydrate and ethanol, but not urethane, alter the clearance of exogenous dopamine recorded by chronoamperometry in striatum of unrestrained rats. Neurosci Lett 343:9-12.

Salgado-Pineda P, Delaveau P, Blin O, and Nieoullon A (2005) Dopaminergic contribution to the regulation of emotional perception. Clin Neuropharmacol 28:228-237.

Scherag S, Hebebrand J, and Hinney A (2010) Eating disorders: the current status of molecular genetic research. Eur Child Adolesc Psychiatry 19:211-226.
Sinclair D, Purves-Tyson TD, Allen KM, and Weickert CS (2014) Impacts of stress and sex hormones on dopamine neurotransmission in the adolescent brain. Psychopharmacology (Berl) 231:1581-1599.

Skarpańska-Stejnborn A, Basta P, Trzeciak J, and Szcześniak-Pilaczyńska Ł (2015) Effect of intense physical exercise on hepcidin levels and selected parameters of iron metabolism in rowing athletes. Eur J Appl Physiol 115:345-351.

Smink FR, van Hoeken D, Oldehinkel AJ, and Hoek HW (2014) Prevalence and severity of DSM-5 eating disorders in a community cohort of adolescents. Int J Eat Disord 47:610-619.

Smith MA, Gergans SR, Iordanou JC, and Lyle MA (2008a) Chronic exercise increases sensitivity to the conditioned rewarding effects of cocaine. Pharmacol Rep 60:561-565.

Smith MA, Schmidt KT, Iordanou JC, and Mustroph ML (2008b) Aerobic exercise decreases the positive-reinforcing effects of cocaine. Drug Alcohol Depend 98:129-135.

Smith MA, Walker KL, Cole KT, and Lang KC (2011) The effects of aerobic exercise on cocaine self-administration in male and female rats. Psychopharmacology (Berl) 218:357-369.

Smith MA and Witte MA (2012) The effects of exercise on cocaine self-administration, food-maintained responding, and locomotor activity in female rats: importance of the temporal relationship between physical activity and initial drug exposure. Exp Clin Psychopharmacol 20:437-446.

Sotak BN, Hnasko TS, Robinson S, Kremer EJ, and Palmiter RD (2005) Dysregulation of dopamine signaling in the dorsal striatum inhibits feeding. Brain Res 1061:88-96.

Symreng T, Cederblad G, Croner S, Larsson J, and Schildt B (1985) Changes in nutritional assessment variables caused by total parenteral nutrition in anorexia nervosa. Clin Nutr 4:81-86.

Tang Y, Qi R, Wu H, Shi W, Xu Y, and Li M (2019) Reduction of hemoglobin, not iron, inhibited maturation of red blood cells in male rats exposed to high intensity endurance exercises. J Trace Elem Med Biol 52:263-269.

Tian Y, Zhao J, Zhao B, Gao Q, Xu J, and Liu D (2012) The ratio of sTfR/ferritin is associated with the expression level of TfR in rat bone marrow cells after endurance exercise. Biol Trace Elem Res 147:261-266.

Tomasi D and Volkow ND (2013) Striatocortical pathway dysfunction in addiction and obesity: differences and similarities. Crit Rev Biochem Mol Biol 48:1-19.

van Gaalen MM, van Koten R, Schoffelmeer AN, and Vanderschuren LJ (2006) Critical involvement of dopaminergic neurotransmission in impulsive decision making. Biol Psychiatry 60:66-73.

Volkow ND, Wang G-J, Maynard L, Jayne M, Fowler JS, Zhu W, Logan J, Gatley SJ, Ding Y-S, Wong C, et al. (2003) Brain dopamine is associated with eating behaviors in humans. Int J Eat Disord 33:136-142.

Willner P (2016) The chronic mild stress (CMS) model of depression: history, evaluation and usage. Neurobiol Stress 6:78-93.

Wojciak R (2014) Alterations of selected iron management parameters and activity in food-restricted female Wistar rats (animal anorexia models). Eat Weight Disord 19:61-68.

Yoshida M, Yokoo H, Mizoguchi K, Kawahara H, Tsuda A, Nishikawa T, and Tanaka M (1992) Eating and drinking cause increased dopamine release in the nucleus accumbens and ventral tegmental area in the rat: measurement by in vivo microdialysis. Neurosci Lett 139:73-76.

Zahniser NR, Larson GA, and Gerhardt GA (1999) In vivo dopamine clearance rate in rat striatum: regulation by extracellular dopamine concentration and dopamine transporter inhibitors. J Pharmacol Exp Ther 289:266-277.

Zipfel S, Giel KE, Bulik CM, Hay P, and Schmidt U (2015) Anorexia nervosa: aetiology, assessment, and treatment. Lancet Psychiatry 2:1099-1111.

Zlebnik NE, Anker JJ, and Carroll ME (2012) Exercise to reduce the escalation of cocaine self-administration in adolescent and adult rats. Psychopharmacology (Berl) 224:387-400

Address correspondence to: Dr. Lynette C. Daws, Department of Cellular and Integrative Physiology, University of Texas Health Science Center at San Antonio, 7703 Floyd Curl Drive, MC7756, San Antonio, TX 78229-3900. E-mail: daws@uthscsa.edu 